\title{
DELEGATION EQUILIBRIUM PAYOFFS IN INTEGER-SPLITTING GAMES
}

\author{
SYLVAIN SORIN AND CHENG WAN
}

\begin{abstract}
This work studies a new strategic game called delegation game. A delegation game is associated to a basic game with a finite number of players where each player has a finite integer weight and her strategy consists in dividing it into several integer parts and assigning each part to one subset of finitely many facilities. In the associated delegation game, a player divides her weight into several integer parts, commits each part to an independent delegate and collects the sum of their payoffs in the basic game played by these delegates. Delegation equilibrium payoffs, consistent delegation equilibrium payoffs and consistent chains inducing these ones in a delegation game are defined. Several examples are provided.
\end{abstract}

AMS Classification: 91A10, 91A18

Keywords: basic integer-splitting game, delegation game, delegation equilibrium payoffs, consistent delegation equilibrium payoffs, consistent chains.

\section{Motivation}

In an $N$-player routing game with parallel paths, each player holds a certain quantity of stock that she has to send by one or several directed paths from its origin vertex to its destination vertex. The cost of using a certain path depends upon the quantity of stock passing through it. Every player wishes to minimize her cost. If the stocks are unsplittable, a player must send all her stock through a single path, otherwise she can split it into several parts so as to send them by different paths. In this work, an integer-splittable case will be considered: every player has a stock of integer weight which can be divided into several parts of integer weight. For example, a player holding a stock of weight 2 can only split it into two parts, each of weight 1 , but not one part of weight 0.5 and another of weight 1.5 . If a player has $R$ available paths, then her pure strategy set is the finite set of the divisions of her stock into $R$ integer weight parts.

Suppose that, instead of deciding by herself how to send her stock, a player $n$ divides it into integer weight parts and commits each part to a different delegate. The delegates are independent players who ensure the transportation of the stocks committed to them. The cost to player $n$ is the sum of the costs to her delegates. This procedure is called (integer) delegation.

Why should a player be interested in delegating her stock? If there is only one player, the minimum cost that she obtains at the equilibrium of this one-player game is just the social optimum cost. If she 
commits her stock to some delegates, then whatever the outcome is, it will not be better than the social optimum. Hence, she has no incentive to delegate.

However, when there are several players, the situation might be different. Here is an example which shows the potential advantage of producing delegates.

Example 1. Consider the following two-player routing game with integer-splittable stock where player $I$ has a stock of weight 2 and player $I I$ has a stock of weight 1 . Both players have to send their stock from vertex $O$ to vertex $D$ in Figure 1. The per-unit cost function of the upper path is $c_{1}(x)=x$, which means that the cost to each unit on the path is $x$ if the total weight is $x$. The per-unit cost of the lower path is $c_{2}(x)=0.1 x+2.3$

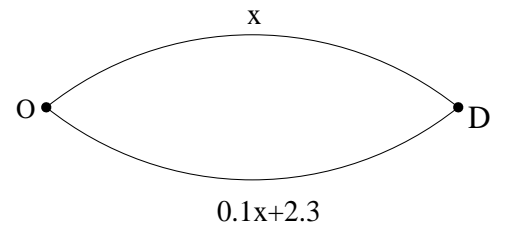

Figure 1.

First, let us consider the routing game. Player $I$ has three pure strategies: $(u, u)$, i.e. to send both units by the upper path, $(u, l)$, i.e. to send one unit by the upper path and the other the lower path, and $(l, l)$, i.e. to send both units by the lower path. Player $I I$ has two pure strategies: $u$, i.e. to send her unit by the upper path, and $l$, i.e. to send it by the lower path. In the cost matrix of the game in Table 1 , player $I$ (resp. player $I I)$ is the row (resp. column) player, and the entries of form $\left(C_{I}, C_{I I}\right)$ are the two players' costs. For example, if player $I$ plays $(u, l)$ and player $I I$ plays $l$, then player $I$ 's cost is 3.5 while player $I I$ 's cost is 2.5. The only pair of pure Nash equilibrium costs $(4.4,2)$ is starred. Besides, as the strategy $(u, l)$ is a dominant one for player $I$, the pure equilibrium is also the unique equilibrium.

\begin{tabular}{r|c|c|}
\multicolumn{1}{c}{$u$} & \multicolumn{1}{c}{$l$} \\
\cline { 2 - 3 }$u, u$ & 6,3 & $4,2.4$ \\
\cline { 2 - 3 }$u, l$ & $4.4,2^{*}$ & $3.5,2.5$ \\
\cline { 2 - 3 }$l, l$ & 5,1 & $5.2,2.6$ \\
\cline { 2 - 3 } & TABLE 1.
\end{tabular}

Now, suppose that player $I$ splits her stock into two parts, each of weight 1 , and commits them to two delegates called $I_{1}$ and $I_{2}$ respectively. In the routing game played by the three players $I_{1}, I_{2}$ and $I I$, each of them has a stock of weight 1 and has two pure strategies $u$ and $l$. The corresponding costs $\left(C_{I_{1}}, C_{I_{2}}, C_{I I}\right)$ are in Table 2. Player $I_{1}$ (resp. player $\left.I_{2}\right)$ is the row (resp. column) player. The left (resp. right) matrix corresponds to the choice $u$ (resp. $l$ ) of player $I I$.

The three pure Nash equilibria are starred. At two of them, $(u, l, u)$ and $(l, u, u)$, player $I$ 's total cost, $C_{I}=C_{I_{1}}+C_{I_{2}}$, and player $I I$ 's cost, $C_{I I}$, are the same as in the previous model without delegation: 4.4 
and 2. At the third one $(u, u, l)$, player I's total cost is 4 and player $I I$ 's cost is 2.4. Player I's cost is lower than in the case without delegation. Conclusion: delegation can be advantageous!

\begin{tabular}{|c|c|c|c|c|c|}
\hline \multicolumn{2}{|r|}{$u$} & $l$ & & $u$ & $l$ \\
\hline$u$ & $3,3,3$ & $2,2.4,2^{*}$ & $u$ & $2,2,2.4^{*}$ & $1,2.5,2.5$ \\
\hline$l$ & $2.4,2,2^{*}$ & $2.5,2.5,1$ & $l$ & $2.5,1,2.5$ & $2.6,2.6,2.6$ \\
\hline
\end{tabular}

TABLE 2 .

Remark 2. Notice that in the three-player game, the equilibrium $(u, u, l)$ exists because player $I I$ is facing the strategy profile $(u, u)$ of the two delegates of player $I$. But if player $I$ knew that player $I I$ would choose $l$, she would not delegate but rather assign by herself one unit weight of stock to $u$ and the other to $l$ so as to get a cost 3.5 instead of 4 . Then the best reply of player $I I$ would be $u$. This underlines the fact that the existence of the equilibria relies on the independence of the delegates.

Since a delegate is an independent player, she may well delegate her stock in her turn. By induction, the procedure can continue until all the players/delegates have a stock of weight 1.

This work will establish a rigorous model of integer-splitting delegation game and define the corresponding delegation equilibrium payoffs.

\section{BAsic (INTEGER-SPlitting) GAME}

2.1. Game definition. Before introducing the notion of delegation, let us first define formally a basic (integer-splitting) game. For example, the routing game with integer-splittable stocks introduced in Example 1 is a basic game.

Definition 3. A basic (integer-splitting) game $G(\mathcal{N}, P)$ is defined by the following elements.

- $Q$ is a nonempty finite set of facilities.

- $P \subset 2^{Q} \backslash\{\emptyset\}$, a collection of nonempty subsets of $Q$, is a nonempty finite set of choices.

- $\mathcal{N}$ is a finite set of players.

A player $n \in \mathcal{N}$ is characterized by three pieces of data:

1) her non zero integer weight $m^{n} \in \mathbb{N}$,

2) the nonempty set of her available choices $P^{n} \subset P$,

3) a vector function $\psi^{n}=\left(\psi_{q}^{n}\right)_{q \in Q}$, where $\psi_{q}^{n}$, her per-unit payoff function for facility $q$, is a realvalued function defined on $[0, M] \cap \mathbb{N}$, where $M=\sum_{n \in \mathcal{N}} m^{n}$.

In particular a player's type is specified by the pair $\left(P^{n}, \psi^{n}\right)$.

- A pure strategy $f^{n}$ of player $n$ is an integer partition of her weight onto $P^{n}$. Explicitly, it is a $\left|P^{n}\right|$-dimensional vector $(|A|$ denote the cardinality of a finite set $A)$ with integer components: 
$f^{n}=\left(f_{p}^{n}\right)_{p \in P^{n}}, f_{p}^{n} \in \mathbb{N}$ being the part of her weight that she assigns to choice $p \in P^{n}$. The finite set of player $n$ 's pure strategies is thus $F^{n}=\left\{f^{n}=\left(f_{p}^{n}\right)_{p \in P^{n}} \in \mathbb{N}^{\left|P^{n}\right|} \mid \sum_{p \in P^{n}} f_{p}^{n}=m^{n}\right\}$.

- $F=\prod_{n \in \mathcal{N}} F^{n}$ is the space of pure-strategy profiles. A profile of pure strategies $f=\left(f^{n}\right)_{n \in \mathcal{N}}$ induces a vector $\xi(f)=\left(\xi_{q}(f)\right)_{q \in Q}$ called aggregated configuration, where $\xi_{q}(f)=\sum_{n \in \mathcal{N}} \sum_{p \in P^{n}: q \in p} f_{p}^{n}$ is the total weight assigned to facility $q$. Denote $X$ the set of feasible aggregated configurations. It is a subset of $\mathbb{N}^{|Q|}$.

- The payoff function of player $n$ is defined as follows:

$$
U^{n}(f)=U^{n}\left(f^{n}, \xi(f)\right)=\sum_{p \in P^{n}} f_{p}^{n} \sum_{q \in p} \psi_{q}^{n}\left(\xi_{q}(f)\right) .
$$

Remark 4. Every player's payoff is additive: the payoff associated to the weight that player $n$ assigns to choice $p$ is counted separately for each choice $p \in P^{n}$, then her total payoff $U^{n}$ is the sum of these payoffs.

Remark 5. A basic game is an anonymous aggregate game according to the definition of $\psi^{n}$ 's. This means that only the total weight assigned to a certain facility is taken into account, but not the identity of the players who send them nor the specific decomposition in terms of choices.

Example 6. When $Q$ is the set of arcs in a finite directed graph, $P$ is the set of elementary directed paths and $P^{n}$ is the set of elementary paths connecting player $n$ 's origin $O^{n}$ to her destination $D^{n}$, the basic game is an extension of Rosenthal's congestion game [6].

The game $G(\mathcal{N}, P)$ is finite: the number of players and the number of strategies for each player is finite. Thus, according to Nash's theorem, there exists an equilibrium (in mixed strategies). The set of equilibrium payoff vectors is denoted by $E(G(\mathcal{N}, P))$. This is a nonempty subset of $\mathbb{R}^{|\mathcal{N}|}$.

In the current analysis, we fix a choice set $P$ and consider different profiles of players with specific available choices in $P$. The set of players of a basic game $G$ is denoted by $\mathcal{N}_{G}$ and, by abuse of notation, $|G|$ will denote its cardinality, i.e. $|G|:=\left|\mathcal{N}_{G}\right|$, the number of players.

2.2. Delegation. Consider a basic game $G$.

Definition 7. A player $n$ in $G$, with weight $m^{n}$ and type $\left(P^{n}, \psi^{n}\right)$, delegates if she is replaced by two players $n^{\prime}$ and $n^{\prime \prime}$ of the same type as her and of strictly positive integer weights $m^{n^{\prime}}$ and $m^{n^{\prime \prime}}$ respectively, and $m^{n^{\prime}}+m^{n^{\prime \prime}}=m^{n}$.

Definition 8. A basic game $G^{\prime}$ is a direct successor of another basic game $G$ if $G^{\prime}$ is obtained by the delegation of one of the players in $G$.

Remark 9. $\left|G^{\prime}\right|=|G|+1$. 
Definition 10. A basic game $G^{\prime}$ is a successor of another basic game $G$ if there exists a finite sequence of basic games $G_{0}, G_{1}, \ldots, G_{k}$ such that $G=G_{0}, G^{\prime}=G_{k}$, and $G_{l}$ is a direct successor of $G_{l-1}$ for $l=1,2, \ldots, k$.

Remark 11. $\left|G^{\prime}\right|=|G|+k$.

Definition 12. Denote the family consisting of a basic game $G$ and all its successors by $\Xi_{G}$. This set has a natural tree structure with root $G$. The derived game $\bar{G}$ of $G$ is the element in $\Xi_{G}$ which is the last on each branch. It has the largest cardinality. In other words, each player in $\bar{G}$ has weight 1, and $|\bar{G}|=M$. Recall that $M=\sum_{n \in \mathcal{N}_{G}} m^{n}$, the total weight of the players in $G$.

\section{EQUILIBRIUM PAYOFFS IN DELEGATION GAMES}

In this section we will define and construct the set of delegation equilibrium payoffs.

3.1. Game form. The game form of the delegation game $\mathcal{D}(G)$ associated to the basic game $G$, i.e. its set of players and their strategy sets, is defined as follows.

The player set is $\mathcal{N}_{G}$, the same as that of $G$.

For each player $n \in \mathcal{N}_{G}$ of weight $m^{n}$, her strategy set $S^{n}$ is the finite set of integer-partitions of $m^{n}$. Explicitly, for each $s^{n} \in S^{n}$, there is an integer $l_{n} \in \mathbb{N}^{*}$ such that

$$
s^{n}=\left\{m_{i}^{n}\right\}_{i=1}^{l_{n}} \in\left(\mathbb{N}^{*}\right)^{l_{n}}, \quad \sum_{i=1}^{l_{n}} m_{i}^{n}=m^{n} .
$$

The interpretation is that player $n$ creates $l_{n}$ delegates with delegate $i$ having weight $m_{i}^{n}, i=1, \cdots, l_{n}$. In particular, the strategy set $S^{n}$ is finite.

Remark 13. A profile of strategies $s=\left\{s^{n}\right\}_{n \in \mathcal{N}}$ induces a successor $G^{\prime}$ of $G$ if there exists $n \in \mathcal{N}$ such that $l_{n}>1$, i.e. at least one player delegates: it is the basic game with player profile defined by $\left\{\left\{m_{i}^{n}\right\}_{i=1}^{l_{n}}\right\}_{n \in \mathcal{N}}$. Otherwise, if $l_{n}=1, s^{n}=\left\{m^{n}\right\}$ for all $n \in \mathcal{N}$, the profile $\left\{s^{n}\right\}_{n \in \mathcal{N}}$ induces the basic game $G$.

3.2. Set of delegation equilibrium payoffs. The payoff functions associated to the delegation game $\mathcal{D}(G)$ and the set of delegation equilibrium payoffs, denoted by $\mathcal{E}(G)$, are defined by induction on the tree $\Xi_{G}$.

For the derived basic game $\bar{G}$, let $\mathcal{E}(\bar{G})$ be $E(\bar{G})$, the set of equilibrium payoffs.

Let $\hat{G} \in \Xi_{G}$ and assume that the set of delegation equilibrium payoffs $\mathcal{E}\left(G^{\prime}\right)$ in $\mathcal{D}\left(G^{\prime}\right)$ is defined for all successors $G^{\prime}$ of $\hat{G}$. We define $\mathcal{E}(\hat{G})$ in three steps.

1) Let $e$ be a selection from $E(\hat{G})$, i.e. $e(\hat{G}) \in E(\hat{G}) \subset \mathbb{R}^{|\hat{G}|}$.

Let $y$ be a selection from the sets of delegation equilibrium payoffs of all successors $\hat{G}$ of $\hat{G}$, i.e. $y\left(G^{\prime}\right) \in$ $\mathcal{E}\left(G^{\prime}\right) \subset \mathbb{R}^{\left|G^{\prime}\right|}$. Let $Y(\hat{G})$ denote the collection of all such selections. 
2) For each pair $(e, y) \in E(\hat{G}) \times Y(\hat{G})$, consider an auxiliary game $\mathcal{G}(\hat{G} ; e, y)$ associated to the game form of $\mathcal{D}(G)$. The payoff $F^{k}$ to player $k \in \mathcal{N}_{\hat{G}}$ as a function of the strategy profile $s=\left\{s^{k}\right\}_{k \in \mathcal{N}_{\hat{G}}}$ is defined as follows.

If $s$ induces the basic game $\hat{G}, F^{k}(s)=e^{k}(\hat{G})$.

If $s$ induces a successor $G^{\prime}$ of $\hat{G}$ so that the player profile of $G^{\prime}$ is $\left\{\left\{m_{i}^{k}\right\}_{i=1}^{l_{k}}\right\}_{k \in \mathcal{N}_{\hat{G}}}$, then

$$
F^{k}(s)=\sum_{i=1}^{l_{k}} y_{i}^{k}\left(G^{\prime}\right)
$$

Namely, the payoff to player $k$ is the sum of the delegation equilibrium payoffs to her delegates according to $y$.

The auxiliary game $\mathcal{G}(\hat{G} ; e, y)$ is a finite game. Therefore, it admits equilibria (in mixed strategies) according to Nash's theorem. We denote by $E(\hat{G} ; e, y) \subset \mathbb{R}^{|\hat{G}|}$ its non empty set of equilibrium payoff profiles.

3) The set of delegation equilibrium payoff profiles in the delegation game $\mathcal{D}(\hat{G})$ is finally defined by

$$
\mathcal{E}(\hat{G})=\bigcup_{e \in E(\hat{G}), y \in Y(\hat{G})} E(\hat{G} ; e, y) .
$$

This ends the construction of $\mathcal{E}$. Hence, we obtain by induction the following:

Proposition 14. The set of delegation equilibrium payoff profiles $\mathcal{E}(G)$ is a nonempty subset of $\mathbb{R}^{|G|}$.

3.3. Consistent delegation equilibrium payoffs. Now we define, for a delegation game $\mathcal{D}(G)$, the set of consistent chains of delegation equilibrium payoffs, denoted by $\mathcal{H}(G) \subset \prod_{G^{\prime} \in \Xi_{G}} \mathbb{R}^{\left|G^{\prime}\right|}$, and the associated set of consistent delegation equilibrium payoffs, denoted by $\tilde{\mathcal{E}}(G) \subset \mathcal{E}(G)$.

The definition of a consistent chain of equilibrium payoffs $h=\left(h\left(G^{\prime}\right)\right)_{G^{\prime} \in \Xi_{G}}$ is obtained by induction as in the previous section.

For $\bar{G}$, let $h(\bar{G})$ be an arbitrary element in $E(\bar{G})=\mathcal{E}(\bar{G})$.

Let $\hat{G} \in \Xi_{G} \backslash\{G\}$ and assume that $h\left(G^{\prime}\right)$ is defined for all successors $G^{\prime}$ of $\hat{G}$ with $h\left(G^{\prime}\right) \in \mathbb{R}^{\left|G^{\prime}\right|}$. As above, for any selection $e$ of $E(\hat{G}) \subset \mathbb{R}^{|\hat{G}|}$, the auxiliary game $\mathcal{G}(\hat{G} ; e, h)$ has a nonempty equilibrium payoff profile set $E(\hat{G} ; e, h) \subset \mathbb{R}^{|\hat{G}|}$. Choose $h(\hat{G})$ as an element in $\bigcup_{e \in E(\hat{G})} E(\hat{G} ; e, h) \subset \mathcal{E}(\hat{G})$. In this way, a consistent chain of delegation equilibrium payoffs $h$ is defined by induction for the delegation game $\mathcal{D}(G)$. Let $\mathcal{H}(G)$ be the collection of all such chains.

For any $h \in \mathcal{H}(G), h(G) \in \mathcal{E}(G)$ is called a consistent delegation equilibrium payoff profile induced by the consistent chain $h$. The set of consistent delegation equilibrium payoff profiles in $\mathcal{D}(G)$, denoted by $\tilde{\mathcal{E}}(G)$, is defined as a subset of $\mathcal{E}(G)$, by

$$
\tilde{\mathcal{E}}(G)=\{h(G)\}_{h \in \mathcal{H}(G)}
$$


The previous induction implies:

Proposition 15. The collection of consistent chains of delegation equilibrium payoffs $\mathcal{H}(G)$ is a nonempty set in $\prod_{G^{\prime} \in \Xi_{G}} \mathbb{R}^{\left|G^{\prime}\right|}$.

The set of consistent delegation equilibrium payoffs $\tilde{\mathcal{E}}(G)$ is a nonempty subset of $\mathcal{E}(G) \subset \mathbb{R}^{|G|}$.

\section{ExAmple}

In this section a delegation game on a network is studied in detail. A delegation equilibrium, a chain of consistent delegation equilibrium payoffs and the consistent delegation equilibrium payoffs that it induces will be obtained. A construction of a non consistent delegation equilibrium profile will also be provided.

The basic game $G$ takes place in a network composed of two vertices $O$ and $D$, with two parallel arcs $r_{1}$ and $r_{2}$ connecting $O$ to $D$. Their per-unit cost functions are respectively $l_{1}(x)=x+1, l_{2}(x)=0.1 x+3.6$. Two players both hold a stock to send from $O$ to $D$. The weight of player $I$ 's stock is 3 and that of player $I I$ 's is 2 . The profile of the players is $\mathcal{N}_{G}=\{I|3, I I| 2\}$, where the name of a player is her type and the number after the name of a player is her weight. $G$ has five successors $A, B, C, D$ and $\bar{G}$. The set of players of the six basic games and some of their equilibrium cost profiles are given below. The type of a player depends on the initial possessor of her stock. In each profile, players of the same type are put in the same pair of brackets.

$$
\begin{array}{ll}
\mathcal{N}_{G}=\{\{I \mid 3\},\{I I \mid 2\}\} & \mathcal{N}_{A}=\left\{\left\{a^{1}\left|2, a^{2}\right| 1\right\},\left\{a^{3} \mid 2\right\}\right\} \\
\mathcal{N}_{B}=\left\{\left\{b^{1}\left|1, b^{2}\right| 1, b^{3} \mid 1\right\},\left\{b^{4} \mid 2\right\}\right\} & \mathcal{N}_{C}=\left\{\left\{c^{1} \mid 3\right\},\left\{c^{2}\left|1, c^{3}\right| 1\right\}\right\} \\
\mathcal{N}_{D}=\left\{\left\{d^{1}\left|2, d^{2}\right| 1\right\},\left\{d^{3}\left|1, d^{4}\right| 1\right\}\right\} & \mathcal{N}_{\bar{G}}=\left\{\left\{q^{1}\left|1, q^{2}\right| 1, q^{3} \mid 1\right\},\left\{q^{4}\left|1, q^{5}\right| 1\right\}\right\} \\
E(G) \supset\{(10.8,6.9),(9.9,7.8)\} & \\
E(A) \supset\{(7.8,3,6.9),(6.9,3,7.8),(6,3.9,7.8),(7.8,3.9,6),(6.9,3.9,6.9)\} \\
E(B) \supset\{(3,3,3.9,7.8),(3,3.9,3,7.8),(3.9,3.9,3.9,6),(3.9,3.9,3,6.9),(3.9,3,3.9,6.9)\} \\
E(C) \supset\{(10.8,3.9,3),(10.8,3,3.9),(9.9,3.9,3.9)\} \\
E(D) \supset\{(7.8,3,3,3.9),(7.8,3.9,3,3),(6,3.9,3.9,3.9),(6.9,3,3.9,3.9),(6.9,3.9,3,3.9)\} \\
E(\bar{G}) \supset\{(3.9,3.9,3.9,3,3),(3.9,3.9,3,3.9,3),(3.9,3,3.9,3.9,3),(3.9,3,3,3.9,3.9),(3,3,3.9,3.9,3.9)\}
\end{array}
$$

Figure 2 illustrates $\Xi_{G}$ by its tree structure.

1) In the delegation game $\mathcal{D}(\bar{G})$, select $h(\bar{G})=(3.9,3.9,3.9,3,3) \in \mathcal{E}(\bar{G})=E(\bar{G})$. Note that another possible selection $y(\bar{G})=(3,3,3.9,3.9,3.9)$.

2) In the basic game $D$, select $e(D)=(7.8,3.9,3,3) \in E(D)$.

3) In the delegation game $\mathcal{D}(D)$, the payoff matrix of the auxiliary game $\mathcal{G}(D ; e, h)$ determined by $(e(D), h(\bar{G}))$ is shown on the left in Table 3, while the one determined by $(e(D), y(\bar{G}))$ is shown on the right in Table 3. Player $d^{1}$ is the row player. Players $d^{2}, d^{3}$ and $d^{4}$ have no actions. 


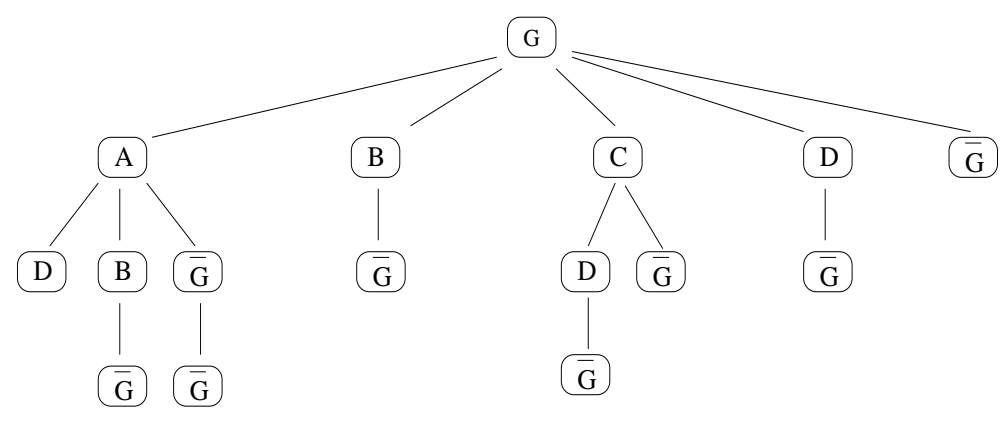

FiguRE 2.

\begin{tabular}{|c|c|c|c|}
\hline$\{2\}$ & $D:(7.8,3.9,3,3) *$ & $\{2\}$ & $D:(7.8,3.9,3,3)$ \\
\hline$\{1,1\}$ & $\bar{G}:(3.9+3.9,3.9,3,3) *$ & $\{1,1\}$ & $\bar{G}:(3+3,3.9,3.9,3.9) *$ \\
\hline
\end{tabular}

The pure equilibria outcomes are starred. In the auxiliary game on the left, select $h(D)=(7.8,3.9,3,3) \in$ $\mathcal{E}(D)$. In the auxiliary game on the right, select $y(D)=(6,3.9,3.9,3.9) \in \mathcal{E}(D)$.

4) In the basic game $C$, select $e(C)=(10.8,3.9,3) \in E(C)$.

$5)$ In the delegation game $\mathcal{D}(C)$, the payoff matrix of the auxiliary game determined by $(e(C), h(D), h(\bar{G}))$ is shown in Table 4. Player $c^{1}$ is the row player. Players $c^{2}$ and $c^{3}$ have no choice.

\begin{tabular}{r|c|}
\cline { 2 - 2 }$\{3\}$ & $C:(10.8,3.9,3)^{*}$ \\
\cline { 2 - 2 }$\{2,1\}$ & $D:(7.8+3.9,3,3)^{*}$ \\
\cline { 2 - 2 }$\{1,1,1\}$ & $\bar{G}:(3.9+3.9+3.9,3,3)^{*}$ \\
& TABLE $4 . \mathcal{G}(C ; e, h)$.
\end{tabular}

Select $h(C)=(10.8,3.9,3) \in \mathcal{E}(C)$.

6) In the basic game $B$, select $e(B)=(3,3.9,3,7.8) \in E(B)$.

7) In the delegation game $\mathcal{D}(B)$, the payoff matrix of the auxiliary game determined by $(e(B), h(\bar{G}))$ is shown in Table 5. Player $b^{4}$ is the row player. Players $b^{1}, b^{2}$ and $b^{3}$ have no action.

$$
\begin{array}{r|c|}
\{2\} & B:(3,3.9,3,7.8) \\
\cline { 2 - 2 }\{1,1\} & \bar{G}:(3.9,3.9,3.9,3+3) * \\
\cline { 2 - 2 } \text { TABLE } 5 . \mathcal{G}(B ; e, h) .
\end{array}
$$

Select $h(B)=(3.9,3.9,3.9,6) \in \mathcal{E}(B)$.

8) In the basic game $A$, select $e(A)=(6.9,3,7.8) \in E(A)$.

9) In the delegation game $\mathcal{D}(A)$, the payoff matrix of the auxiliary game determined by $(e(A), h(D), h(B)$, $h(\bar{G}))$ is shown in Table 6 . Player $a^{1}$ is the row player and player $a^{3}$ is the column player. Player $a^{2}$ has no action.

Select $h(A)=(7.8,3.9,6) \in \mathcal{E}(A)$. 
$\{2\}$

$\{1,1\}$

\begin{tabular}{|c|c|c|}
\hline$\{2\}$ & $A:(6.9,3,7.8)$ & $D:(7.8,3.9,3+3) *$ \\
\hline 1,1$\}$ & $B:(3.9+3.9,3.9,6)$ & $\bar{G}:(3.9+3.9,3.9,3+3)^{*}$ \\
\hline
\end{tabular}

10) In the basic game $G$, select $e(G)=(9.9,7.8) \in E(G)$.

11) In the delegation game $\mathcal{D}(G)$, the payoff matrix of the auxiliary game determined by $(e(G), h(A), h(B)$, $h(C), h(D), h(\bar{G}))$ is shown in Table 7. Player $I$ is the row player and player $I I$ is the column player.

\begin{tabular}{r|c|c|}
\multicolumn{1}{c}{2} & $\{1,1\}$ \\
\cline { 2 - 3 }$\{3\}$ & $G:(9.9,7.8)$ & $C:(10.8,3.9+3)^{*}$ \\
\cline { 2 - 3 }$\{2,1\}$ & $A:(7.8+3.9,6)$ & $D:(7.8+3.9,3+3)$ \\
\cline { 2 - 3 }$\{1,1,1\}$ & $B:(3.9+3.9+3.9,6)$ & $\bar{G}:(3.9+3.9+3.9,3+3)$ \\
\cline { 2 - 3 } & \multicolumn{2}{|c}{ TABLE $7 . \mathcal{G}(G ; e, h)}$.
\end{tabular}

Select $h(G)=(10.8,6.9)$. Then the construction of a consistent chain of delegation equilibrium profiles $h$ is completed, and $(10.8,6.9)$ is a consistent delegation equilibrium profile in $\mathcal{D}(G)$.

Alternatively, the auxiliary game $\mathcal{G}(G ; e, y)$ which uses $y(D)$ instead of $h(D)$ has the payoff matrix in Table 8.

\begin{tabular}{r|c|c|}
\multicolumn{1}{c}{2} & $\{1,1\}$ \\
\cline { 2 - 3 }$\{3\}$ & $G:(9.9,7.8)$ & $C:(10.8,3.9+3)$ \\
\cline { 2 - 3 }$\{2,1\}$ & $A:(7.8+3.9,6)$ & $D:(6+3.9,3.9+3.9)$ \\
\cline { 2 - 3 }$\{1,1,1\}$ & $B:(3.9+3.9+3.9,6)$ & $\bar{G}:(3.9+3.9+3.9,3+3)$ \\
\cline { 2 - 3 } & \multicolumn{2}{|c|}{ TABLE 8. $\mathcal{G}(G ; e, y)}$.
\end{tabular}

The only equilibrium is attained if player $I$ plays $\{3\}$ with probability $2 / 3$ and $\{2,1\}$ with probability $\frac{1}{3}$, and player $I I$ plays $\{2\}$ with probability $1 / 3$ and $\{1,1\}$ with probability $\frac{2}{3}$. The equilibrium cost profile is $(10.5,7.2)$. This is an equilibrium cost profile in $\mathcal{D}(G)$ but is not constructed by a consistent chain.

\section{Comments And extensions}

5.1. Nash equilibria. One could define equilibrium payoffs $F(G)$ in the delegation game $\mathcal{D}(G)$ by induction using first an auxiliary game $\mathcal{G}(G ; e, z)$ where $e$ is a selection of $E(G)$ and for each successor $G^{\prime}$ and $z\left(G^{\prime}\right)$ is a selection of feasible payoff in $G^{\prime}$. Then, one would require that an equilibrium $\sigma$ in the auxiliary game satisfies the coherency condition: for each $G^{\prime}$ in the support of $\sigma, z\left(G^{\prime}\right)$ belongs to $F\left(G^{\prime}\right)$. However, this would assume that off the equilibrium path the delegate players would play a specific "threat" which is not an equilibrium and is thus difficult to justify. 
5.2. Subgame perfection. The induction procedure used to define delegation equilibria is reminiscent of subgame perfection [7]. However, there are important differences. First, the tree structure involves new players and the consistency argument used in subgame perfection (the (same) players should play an equilibrium at each node of the tree) is replaced by an argument involving the rationality of the delegates. Moreover, for the notion of consistent delegation equilibrium, one goes even one step further since one asks for the same perception of the play in the successor games. One could relate the construction of delegation equilibrium to the usual selection of subgame perfect equilibria through forward induction: if $G^{\prime}$ is reached, play an equilibrium in $G^{\prime}$. Then, the notion of consistent delegation equilibria would add a backward induction property: the behavior in a successor game $G^{\prime}$ of $G$ is independent of the way $G^{\prime}$ is reached during the hypothetical play of $\mathcal{D}(G)$ and depends only on $G^{\prime}$ (and its successors).

5.3. Composite equilibria. The same concepts can be defined in games where each player has a finitely divisible stock, even in the presence of a set of nonatomic players who hold each an infinitesimal stock, using the notion of composite equilibria [3, 8]. However, the general case where each player $n$ can divide arbitrarily her stock $m^{n}$ composed of finitely (or countably) many atoms $m_{i}^{n}, i \geq 1$ and a nonatomic part $m_{0}^{n}$ with $\sum_{j=0}^{+\infty} m_{j}^{n}=m^{n}$ deserves further study, since the definition of delegation equilibrium by backward induction is no longer available.

5.4. A special case. A special case where there are only two choices in the basic game is studied in $[9$, Chapter 3]. In such a setting, all players have the same payoff function. In the basic game $G$, a player holding a stock of strictly positive weight can arbitrarily divide her stock into two parts and affect them to the two choices respectively and is called atomic splittable. Under a standard concavity assumption on the payoff functions, a unique equilibrium exists in each basic game. In a delegation game, each atomic splittable player can arbitrarily delegate her stock to finitely many atomic delegates and a set of nonatomic delegates. Her payoff is the sum of the payoffs to her delegates at the equilibrium of the basic game played by all the delegates. It is shown that, if one or several players delegate, then the payoff to any player not delegating is reduced or does not change in any successor $G^{\prime}$ of $G$ compared with $G$, and the social payoff, i.e. the sum of the payoffs to all the players, is reduced or does not change in $G^{\prime}$ compared with $G$.

5.5. Final comments. Several papers (for example, $[4,2,1]$ ) deal with variation of the structure of players in congestion games, in particular, comparing the social optimum cost to the cost at the Wardrop equilibrium [10]. Other approaches (for example, [8]) evaluate the "advantage of coalitions". In all such frameworks, a procedure has to be defined that allows for such a cooperative behavior.

In our case, - and this is specific to these anonymous games - the fact that one player delegates is: 1) feasible in terms of strategies, and 2) undetectable to the other players. This appears then as a true 
strategic opportunity that has to be taken into account. This approach seems to be new and would lead to an alternative evaluation of "price of competition".

Acknowledgments. We thank G. Vigeral and the anonymous referee for their valuable comments and suggestions.

\section{REFERENCES}

[1] U. Bhaskar, L. Fleischer and C.-C. Huang, The price of collusion in series-parallel networks, in Integer Programming and Combinatorial Optimization, edited by F. Eisenbrand and F.B. Shepherd. Springer Berlin Heidelberg (2010) 313-326.

[2] R. Cominetti, J.R. Correa and N.E. Stier-Moses, The impact of oligopolistic competition in networks. Oper. Res. 57 (2009) 1421-1437.

[3] P.T. Harker, Multiple equilibrium behaviors on networks. Transportation Sci. 22 (1988) 39-46.

[4] A. Hayrapetyan, É. Tardos and T. Wexler, The effect of collusion in congestion games (extended abstract), in Proc. of the 38th Annual ACM Symposium on Theory of Computing, edited by J.M. Kleinberg. ACM, New York (2006) 89-98.

[5] C.-C. Huang, Collusion in atomic splittable routing games, in Proc. of the 38th International Conference on Automata, Languages and Programming, edited by L. Aceto, M. Henzinger and J. Sgall. Springer Berlin Heidelberg (2011) 564-575.

[6] R.W. Rosenthal, A class of games possessing pure-strategy Nash equilibria. Int. J. Game Theory 2 (1973) 65-67.

[7] R. Selten, A reexamination of the perfectness concept for equilibrium points in extensive games. Int. J. Game Theory 4 (1973) 25-55.

[8] C. Wan, Coalitions in network congestion games. Math. Oper. Res. Online first: June 28, 2012. doi: 10.1287/moor.1120.0552.

[9] C. Wan, Contributions à la théorie des jeux d'évolution et de congestion. Ph.D. thesis, Université Pierre et Marie Curie (Paris 6), France (2012).

[10] G. Wardrop, Some theoretical aspects of road traffic research communication networks, in Proc. Inst. Civ. Eng. II(1) (1952) 325-378.

Sylvain Sorin, Combinatoire et Optimisation, IMJ, CNRS UMR 7586, Faculté de Mathématiques, Université P. et M. Curie - Paris 6, Tour 15-16, 1Ère Étage, 4 Place Jussieu, 75005 Paris and Laboratoire d’Econométrie, Ecole Polytechnique, France

$$
\text { E-mail address: sorin@math.jussieu.fr }
$$

http://www.math.jussieu.fr/ sorin/

Cheng Wan, Combinatoire et Optimisation, imJ, CNRS UMR 7586, Faculté de Mathématiques, Université P. et M. Curie - Paris 6, Tour 15-16, 1Ère Étage, 4 Place Jussieu, 75005 Paris

E-mail address: cheng.wan.2005@polytechnique.org

https://sites.google.com/site/wancheng2012avril/ 